Axions from cosmic string and wall decay

C. A. Hagmann

March 11, 2010

Axions 2010

Gainesville, FL, United States

January 15, 2010 through January 17, 2010 
This document was prepared as an account of work sponsored by an agency of the United States government. Neither the United States government nor Lawrence Livermore National Security, LLC, nor any of their employees makes any warranty, expressed or implied, or assumes any legal liability or responsibility for the accuracy, completeness, or usefulness of any information, apparatus, product, or process disclosed, or represents that its use would not infringe privately owned rights. Reference herein to any specific commercial product, process, or service by trade name, trademark, manufacturer, or otherwise does not necessarily constitute or imply its endorsement, recommendation, or favoring by the United States government or Lawrence Livermore National Security, LLC. The views and opinions of authors expressed herein do not necessarily state or reflect those of the United States government or Lawrence Livermore National Security, LLC, and shall not be used for advertising or product endorsement purposes. 


\title{
Axions from cosmic string and wall decay
}

\author{
Chris Hagmann ${ }^{\mathrm{a}}$ \\ ${ }^{a}$ Lawrence Livermore National Laboratory, L-59, 7000 East Ave, Livermore, CA
}

\begin{abstract}
If inflation occurred with a reheat temperature $>T_{\mathrm{PQ}}$, axions from the decay of global axion strings and domain walls would make an important contribution to the cosmological energy density, comparable to that from vacuum misalignment. Several groups have numerically studied the evolution of axion strings and walls in the past, however substantial uncertainties remain in their contribution to the present density $\Omega_{\mathrm{a} \text {,string+wall }} \sim 1-100$ $\left(f_{a} / 10^{12} \mathrm{GeV}\right)^{7 / 6}$, where $f_{a}$ is the axion decay constant. I will describe the numerical methods used in our simulations and show results for several string and wall configurations.
\end{abstract}

Keywords: axion, axion string, axion domain wall.

PACS: $14.80 . \mathrm{Va}$

\section{INTRODUCTION}

Global axion strings are produced in the early universe due to the spontaneous breaking of the global $\mathrm{U}(1)_{\mathrm{PQ}}$ symmetry [1] at the energy scale $f_{a}$, with the axion being the associated Goldstone boson [2]. The phase of the complex PQ scalar field and hence the axion field needs to be continuous in space. Any closed path over which the phase changes by $2 \pi$ must necessarily contain a string core of size $1 / f_{a}$ where the axion field vanishes. The strings have an energy per unit length of $\mu=\pi f_{a}^{2} \ln \left(R f_{a}\right)$, where $R$ is the long range cutoff given by the inter-string distance. The tension in a bent string will cause it to oscillate and radiate axions in analogy to photons emitted by an accelerating electric charge. Crossing strings frequently intercommute [3] and form loops which subsequently collapse. As time increases more long strings will enter the horizon but through the process of loop formation and decay, a stable scaling solution for the network will be reached quickly, with the energy density in long strings at time $t$ given by

$$
\rho_{\mathrm{s}}(t)=\xi \frac{\mu(t)}{t^{2}}
$$

where $\xi$ is a constant of order unity. When the temperature reaches $T_{\mathrm{QCD}} \sim 1 \mathrm{GeV}$, the axion mass turns on and the Mexican hat potential develops a slight tilt. This causes axion domain walls with areal energy density $\sim m_{a} f_{a}^{2}$ to form between string cores. The wall tension rapidly pulls the strings together leading to the demise of the stringwall network. In addition to axions from string and wall decay there will a third source of non-thermal axions due to vacuum misalignment in spatial regions void of topological defects. The former two contributions will be absent if inflation occurs with a reheat temperature $T_{\text {reheat }}<T_{\mathrm{PQ}}$ leading to an initially homogenous axion field. In the opposite case with $T_{\text {reheat }}>T_{\mathrm{PQ}}$, the contributions from string and wall decay to 
the present axion energy density depend crucially on the axion radiation spectrum. The reason for this is that the majority of radiated axions become non-relativistic shortly after $T_{\mathrm{QCD}}$ and the number density is the important quantity for estimating $\Omega_{a}$. Two extreme scenarios have been put forth in the literature: (A) loops are weakly damped and the spectrum is peaked at a wavelength of order the loop size [4-7], and (B) loops are critically damped and radiate axions with a $1 / k$ spectrum [8-11]. It is useful to define the quantity

$$
N_{a x}(t) \equiv \int d k \frac{d E}{d k}(t) \frac{1}{k}
$$

where $k$ is the wave vector and $d E / d k$ is the axion spectrum, both due to the self-field and radiated axions. For a collapsing loop, $r \equiv N_{a x}^{r a d} / N_{a x}^{\text {self }}$ is thus a measure of the hardening of the spectrum relative to the initial $1 / k$ self-field spectrum. An additional unknown parameter of the scaling solution is the average initial loop size at time $t$ defined here as $\chi \equiv t / \bar{R}$. The present axion density from string decay can be expressed as [9]

$$
\Omega_{a, \text { string }} \cong \frac{\xi \bar{r}}{\chi} \Omega_{a, \text { mis }} \cong 0.35 \frac{\xi \bar{r}}{\chi}\left(\frac{f_{a}}{10^{12} \mathrm{GeV}}\right)^{7 / 6}\left(\frac{0.7}{h}\right)^{2},
$$

where $h$ is the Hubble parameter in units of $100 \mathrm{~km} \mathrm{~s}^{-1} \mathrm{Mpc}^{-1}$ and $\Omega_{a, m i s}$ is the contribution from vacuum misalignment for $T_{\text {reheat }}>T_{\mathrm{PQ}}$. There is considerable controversy in the literature over the correct values of $\xi, \bar{r}$, and $\chi$. The results derived for local strings are not applicable here, since axion strings are global defects. At present, numerical simulations are the most promising tool for pinning down the parameters of the axion string network. A similar situation exists for domain walls with [10]

$$
\Omega_{a, \text { wall }} \cong \frac{6}{\gamma} \Omega_{a, \text { mis }} \cong \frac{2.1}{\gamma}\left(\frac{f_{a}}{10^{12} \mathrm{GeV}}\right)^{7 / 6}\left(\frac{0.7}{h}\right)^{2},
$$

where $\gamma \equiv \frac{\varpi}{m_{a}}$ is the average Lorentz factor of the axions radiated during wall collapse.

\section{STRING SIMULATIONS}

Our group has studied the following isolated systems: (1) collapsing circular loops, (2) rotating loops, (3) bent strings, and (4) rotating string-antistring pairs [9]. The equations of motion in difference form on a fixed Cartesian grid were used to evolve the complex scalar field in time. Initial configurations were relaxed with the cores held 


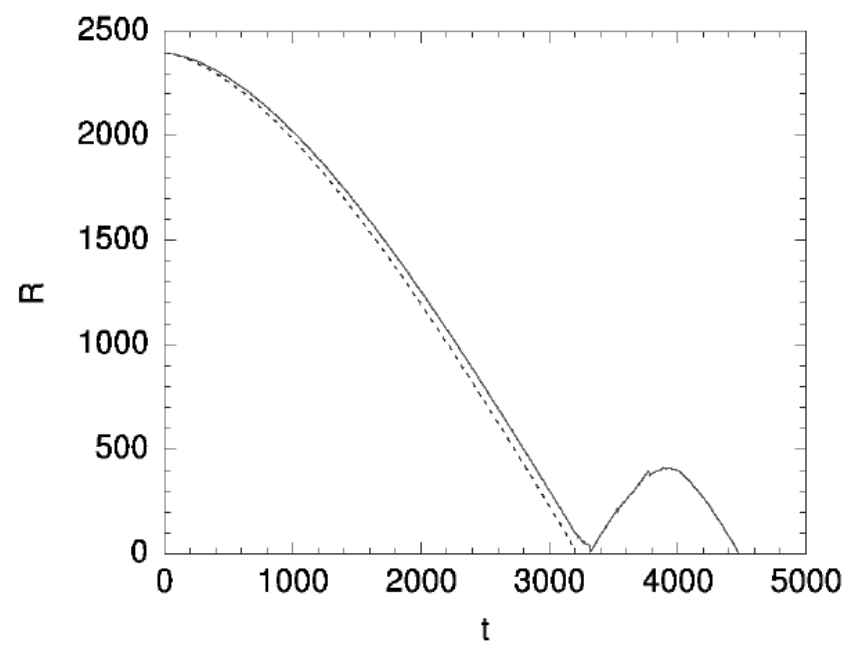

FIGURE 1. Core radius versus time of a collapsing circular loop for $\delta=32$ (dotted line) and $\delta=16$ (solid line).

fixed and spectrum analysis of the total axion field was performed at fixed time intervals during evolution. Fig. 1 shows the collapse of a circular loop initially at rest for different values of $\delta$. To achieve large values of $\ln \left(R_{0} / \delta\right) \sim 5$ the simulations were done in $2 \mathrm{D}$ by exploiting the symmetry of the problem. The minimum core thickness was dictated by the need to resolve the highly contracted core during the final stage of collapse. Fig. 2 depicts the quantity $N_{a x}$ as a function of time for various values of $\delta$, with $r$ decreasing by about $80 \%$ for all cases studied.

More general non-circular loops were also simulated. The initial configuration of the string core was a non-intersecting Kibble-Turok loop [12] with angular momentum. They exhibited the same non-oscillatory behavior as circular loops with a small decrease in $N_{a x}$ during collapse for $\ln \left(R_{0} / \delta\right) \sim 3$.

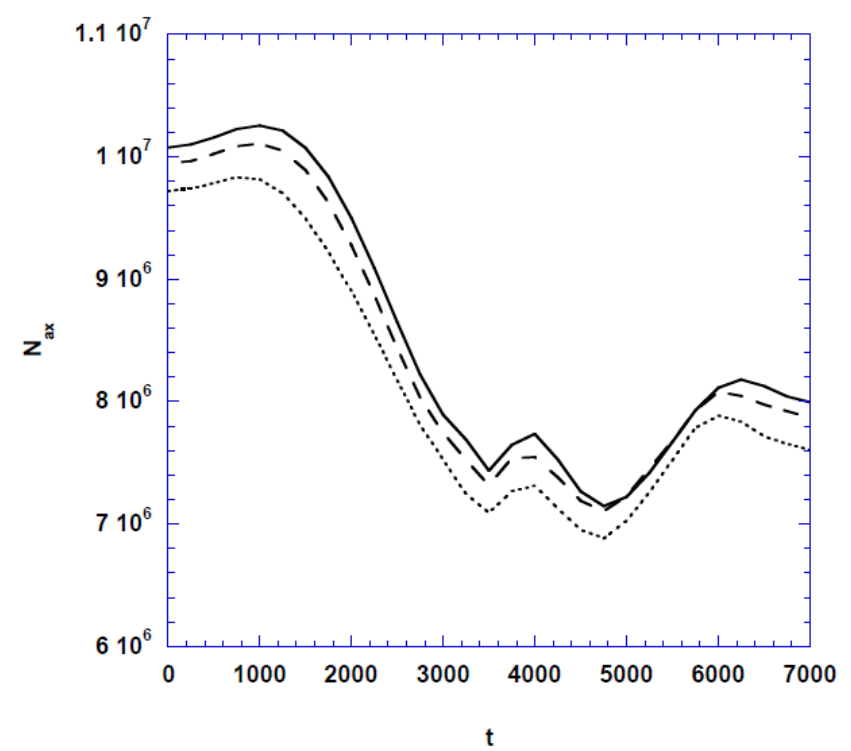

FIGURE 2. $N_{a x}$ versus time of a circular loop for $R_{0}=2400, L_{\rho}=4000, L_{z}=4096$ and $\delta=16$ (solid), $\delta=32$ (dashed), and $\delta=63$ (dotted). 


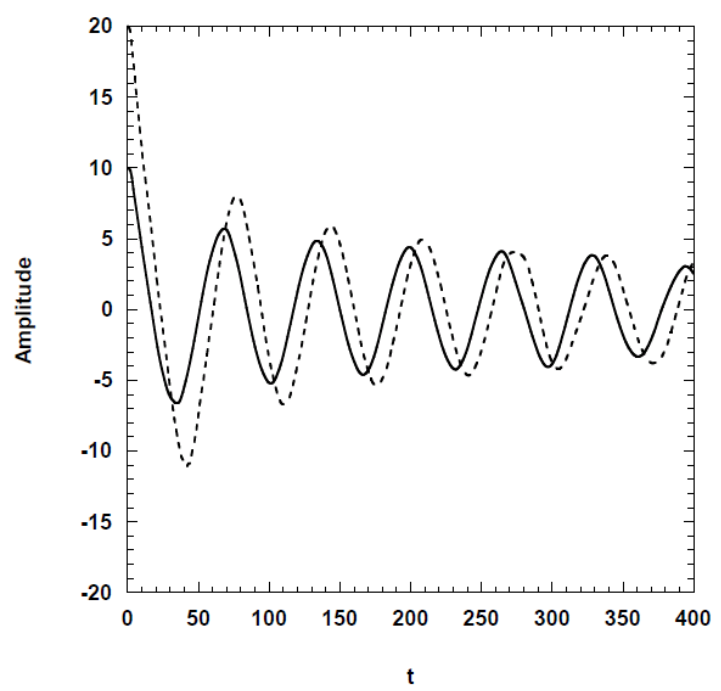

FIGURE 3. String core position of oscillating bent strings versus time. The simulation is carried out on a $L_{x} L_{y} L_{z}=256^{2} * 64$ lattice with a wavelength of 64 lattice units, and $\delta=2.2$.

We also carried out simulations of bent oscillating strings with their ends held fixed. Qualitatively, the motion can be described as follows. If the initial amplitude is larger than about $10 \%$ of the wavelength, the string is strongly damped. For smaller amplitudes the string appears more weakly damped. In all cases the energy released as radiation was always small compared to the self-field energy and $N_{a x}$ changed little during evolution. Finally we studied the annihilation of rotating string-antistring pairs in 2D. The Abrikosov ansatz was used to initialize the axion field followed by relaxation. Again we observed a decreasing $r$ for all parameters investigated.

\section{WALL SIMULATIONS}

The decay of isolated domain walls bounded by strings was simulated to obtain the spectrum of radiated axions [10]. Straight domain walls were set up on a 2D static grid with $f_{a} / m_{a}<50$ while the cosmologically relevant scale is $10^{26}$. The walls were initialized with the sine-Gordon ansatz and held fixed during relaxation. If angular momentum was nonzero, the initial time derivative of the field was obtained as the difference of two relaxed wall configurations a small time step apart A first order in time, and second order in space algorithm was used for the dynamical evolution with $d t=0.2$. The boundary conditions were periodic throughout and the total energy was conserved to better than $1 \%$. The evolution of the domain wall was studied for various values of $f_{a} / m_{a}$, the initial wall length $D$ and the initial velocity $v$ of the strings in the direction transverse to the wall, the string and antistring going in opposite directions. The strings attached to the wall are rapidly accelerated by the wall tension, the potential and gradient energies of the wall being converted into kinetic energy of the strings. The field configuration during this evolution is shown in Fig. 4. For a domain 


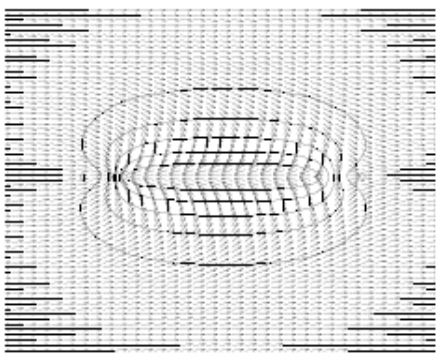

(a)

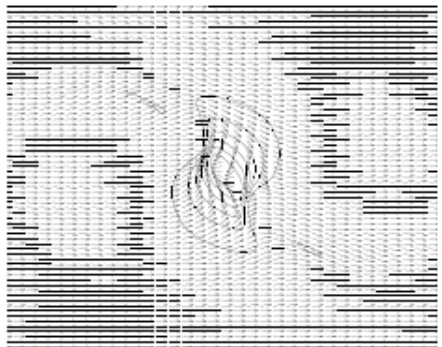

(c)

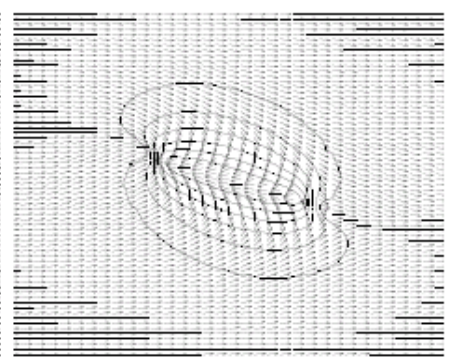

(b)

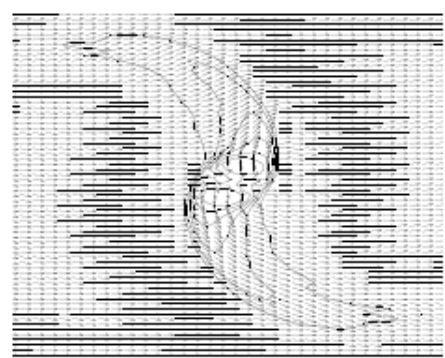

(d)

FIGURE 4. Decay of a wall at successive time intervals $\Delta t=1.2 / \mathrm{m}_{a}$ for the case $D=524,1 / \mathrm{m}_{a}=100$, $\delta=10$, and $v(0)=0.6$.

wall without rotation, the string cores meet head-on and go through each other and regenerate a wall of reduced length. We performed spectrum analysis of the axion field as a function of time to compute the average $\gamma$ factor which is plotted in Fig. 5 for various values of $f_{a} / m_{a}$. An extrapolation to $f_{d} / m_{a}=10^{26}$ yields $\gamma \sim 60$.

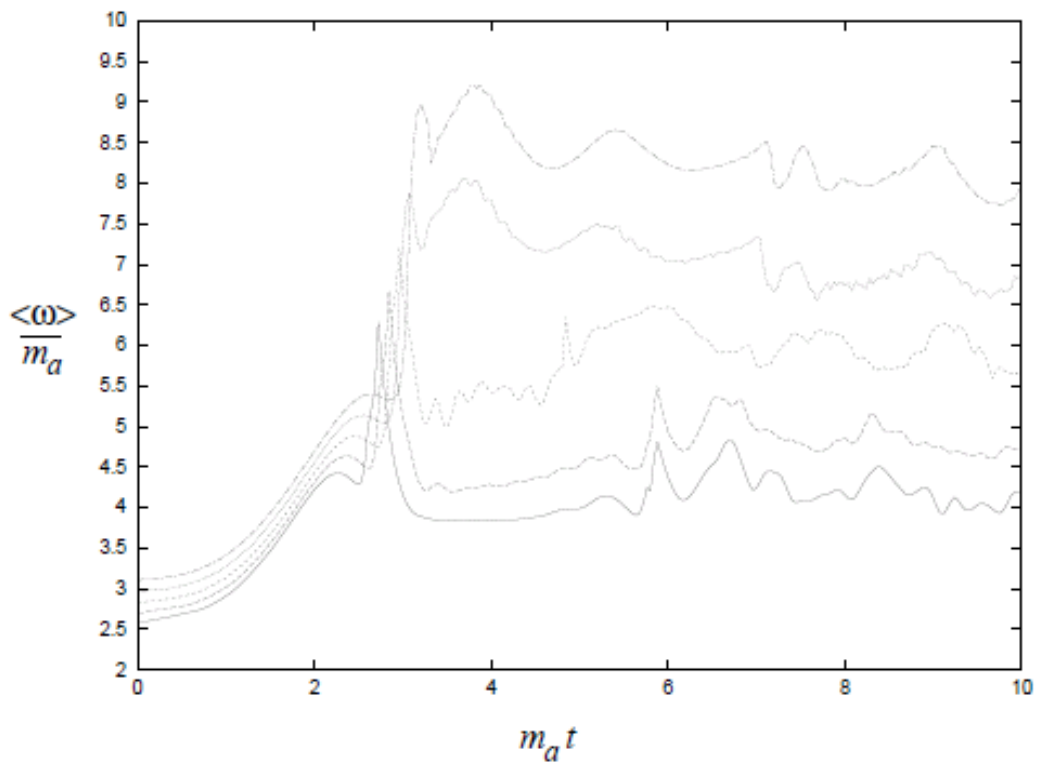

FIGURE 5. Average axion energy as a function of time for a collapsing domain wall with $D=2096$, $1 / m_{a}=500$, and $v(0)=0.25$. The curves are computed for wall to string core ratios of $10,14.1,20,28.3$, and 40 (from bottom to top). 


\section{CONCLUSION AND OUTLOOK}

We have presented the results of our simulations of the decay of axion strings and axion walls bounded by strings. Our emphasis has been on calculating the spectral hardening parameter $\bar{r}$ in the case of strings and the average energy $\gamma$ of emitted axions in the case of walls. We have only an estimate for $\chi \sim 1$, and adopted $\xi \sim 1$ from Yamaguchi and coworkers [7]. Shellard et al. [6] on the other hand have consistently used $\xi \sim 13$ originally derived for local strings. There is also considerable disagreement in the literature on the radiation spectrum from strings. Two groups [6-7] argue the spectrum is peaked at a wavelength of order the loop size whereas our simulations support a $1 / k$ spectrum. With regard to wall decay, our results based on a large extrapolation indicate $\gamma \sim 60$ while Nagasawa et al. [13] obtained $\gamma \sim 3$. Plugging the different parameters into Equations (3) and (4) gives a rather large range $\Omega_{\text {a,string+wall }} \sim 1-100\left(f_{a} / 10^{12} \mathrm{GeV}\right)^{7 / 6}$. Clearly a more definitive calculation is highly desirable. Methods using fixed meshes might reach $\ln (R / \delta) \sim 10$ on highly parallel machines whereas the interesting range is $\ln (R / \delta) \sim 60-70$. A possible alternative might be the use of adaptive mesh methods to achieve the needed dynamic range.

\section{ACKNOWLEDGMENTS}

This work was performed under the auspices of the U.S. Department of Energy by Lawrence Livermore National Laboratory under Contract DE-AC52-07NA27344.

\section{REFERENCES}

1. R. Peccei and H. Quinn, Phys. Rev. Lett. 38, 1440 (1977); Phys. Rev. D16, 1791 (1977).

2. S. Weinberg, Phys. Rev. Lett. 40, 223 (1978); F. Wilczek, Phys. Rev. Lett. 40, 279 (1978).

3. E.P.S. Shellard, Nucl. Phys. B283, 624 (1987).

4. R. Davis, Phys. Rev. D32, 3172 (1985); Phys. Lett. B180, 225 (1986).

5. R.L. Davis and E.P.S. Shellard, Nucl. Phys. B324, 167 (1989).

6. R.A. Battye and E.P.S. Shellard, Nucl. Phys. B423, 260 (1994); Phys. Rev. Lett. 73, 2954 (1994); (E) ibid. 76, 2203 (1996).

7. M. Yamaguchi, M. Kawasaki, and J. Yokoyama, Phys. Rev. Lett. 82, 4578 (1999); M. Yamaguchi, Phys. Rev. D60, 103511 (1999); M. Yamaguchi, J. Yokoyama, and M. Kawasaki, ibid. D61, 061301 (R) (2000).

8. D. Harari and P. Sikivie, Phys. Lett. B195, 361 (1987).

9. C. Hagmann, S. Chang, and P. Sikivie, Phys. Rev. D63, 125018 (2001).

10. S. Chang, C. Hagmann, and P. Sikivie, Phys. Rev. D59, 023505 (1999).

11. P. Sikivie, Lect. Notes Phys. 741, 19 (2008).

12. T.W.B. Kibble and N. Turok, Phys. Lett. B116, 141 (1982); A.L. Chen, D.A. DiCarlo, and S.A. Hotes, Phys. Rev. D37, 863 (1988).

13. Nagasawa and M. Kawasaki, Phys. Rev. D50, 4821 (1994). 